\title{
Baetis punicus n. sp., Ephéméroptère nouveau de Tunisie (Baetidae)
}

\author{
A.G.B. Thomas 1 \\ M. Boumaiza2 \\ T. Soldán ${ }^{3}$
}

Description illustrée de la larve (au dernier stade) de Baetis punicus n. sp. Cette espèce, rencontrée dans le Nord-Ouest de la Tunisie, appartient au groupe alpints : elle est comparée à cette dernière espèce et à $B$. maurus Kimmins.

Baetis punicus n. sp., a new mayfly from Tunisla (Ephemeroptera, Baetidae).

The nymph (last instar) of Baetis punicus n. sp., from north-western Tunisia, is described and illustrated. This species belongs to the alpinus group: it is compared to this latter species and to B. maurus Kimmins.

L'étude faunistique de plusieurs réseaux hydrographiques tunisiens a permis la récolte de larves d'une espèce nouvelle de Baetis appartenant au groupe alpinus. Les imagos et subimagos sont encore inconnues.

\section{1. - Baetis punicus n. sp. : description}

Larve au dernier stade.

Diagnose sommaire : espèce de taille moyenne, à la coloration brun jaunâtre clair, plutôt uniforme. Paracerque court, de longueur variable mais approximativement comprise entre la largeur du $8^{\text {e }}$ tergite et celle du $10^{e}$.

TETE.

Traces d'insertions musculaires du crâne assez apparentes, surtout chez la . .

Antennes: le scape et le pédicelle, dépourvus d'écailles, portent seulement de courtes soies.

Labre (fig. 1) : il a une morphologie voisine de celle de $B$. alpinus, c'est-à-dire un rapport largeur/lon-

1. Laboratoire d'Hydrobiologie, ERA 702 du C.N.R.S. U.P.S., 118 route de Narbonne. F-31062 Toulouse Cedex. France.

2. Laboratoire de Biologie Animale. Faculté des Sciences, Campus Universitaire El Menzah, Tunis. Tunisie.

3. Institute of Entomology, Czechoslovak Academy of Sciences. Na Sádkách 702. CS 370 os Ceské Budejovice, Czechoslovakia. gueur - soies bordantes exclues - compris entre 1,6 et 1,7 et une depression médiodistale bien visible. Le nombre des grandes soies par demi-région frontale est cependant plus faible : $1+11-12$, mais pouvant varier entre 10 et 14 . Ces soies, relativement fines (diamètre à la base d'environ 3 à $3,5 \mu \mathrm{m})$, sont insérées selon une ligne légèrement convexe vers l'avant.

Maxilles : les palpes portent, à l'apex, des écailles implantées dans des alvéoles, le plus souvent au nombre de 2 ou 3, parfois une seule ou même aucune (fig. 2). Ces écailles, en général plus courtes que chez $B$. alpinus, peuvent être remplacées par de simples épaississements chitineux en forme de dent.

Mandibules (fig. 3) pratiquement identiques à celles de B. alpinus, avec une assez forte asymétrie des prosthecas.

Labium : apex des palpes (fig. 4) un peu plus étroit et allongé, un peu moins arrondi et moins massif que chez B. alpinus. Ce contour est très proche de celui de B. nicolae Thomas (voir Thomas et Gazagnes 1983, fig. 5]. Paraglosses, fig. 5.

\section{THORAX.}

Dessin des insertions musculaires du pronotum très voisin de celui de $B$. alpinus, mais moins contrasté. La couleur de fond du pronotum est, elle aussi, plutôt claire. 

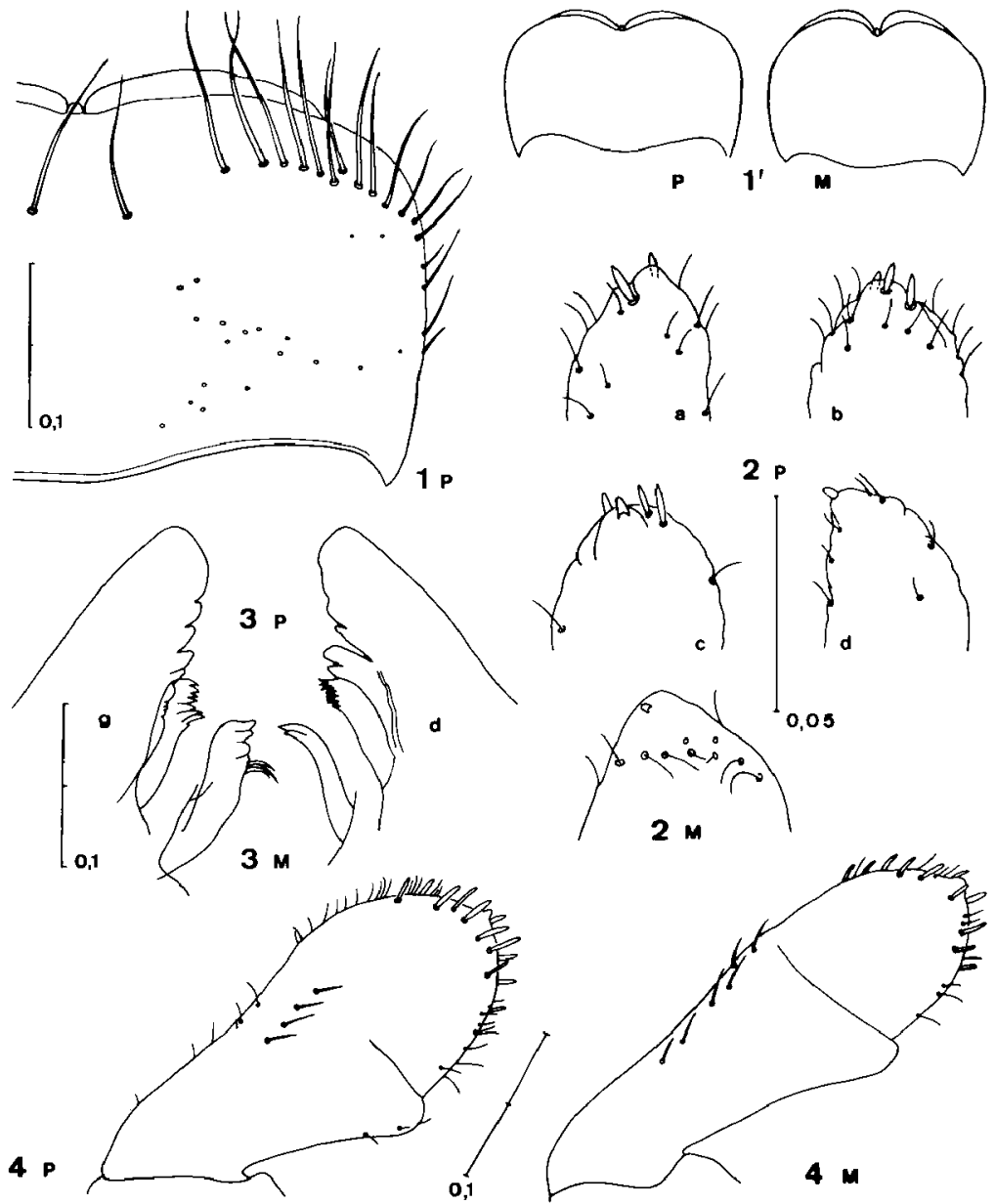

Fig. 1 à 4. Larves de Baetis au dernier stade : $\mathbf{P}=$ B. punicus $\mathrm{n}$. sp.; $\mathbf{M}=$ B. maurus Kimmins. Echelle en mm. 1 : détail du labre - 1' : forme générale - $2:$ apex du palpe maxillaire (a et $b$ : holotype) - $3:$ apex et prostheca des mandibules droite (d) et gauche (g) $-4: 2^{e}$ et $3^{e}$ articles du palpe labial, face dorsale. 


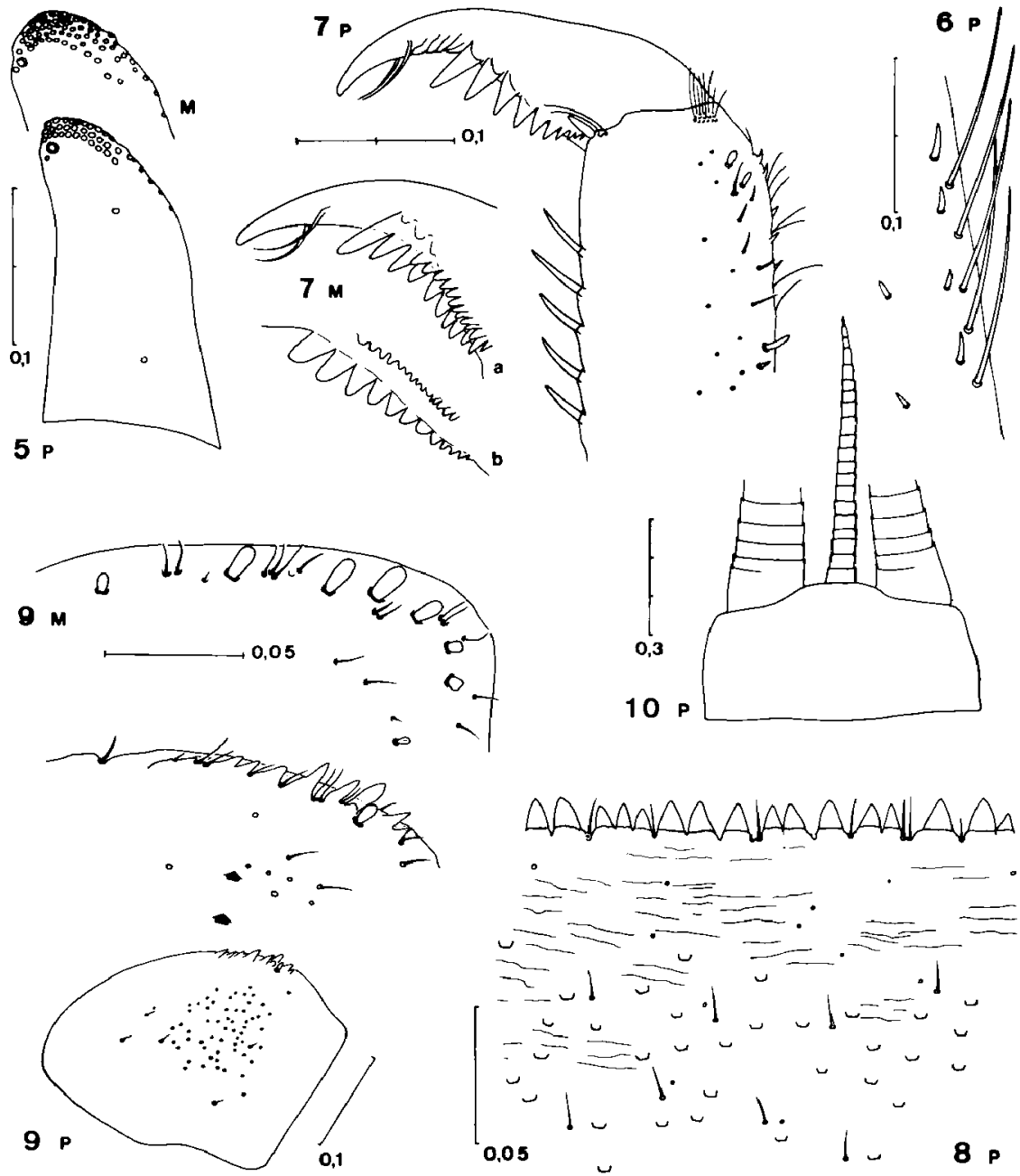

Fig. 5 à 10. Larves de Baetis au dernier stade : $\mathbf{P}=$ B. punicus n. sp. ; $\mathbf{M}=$ B. maurus Kimmins. Echelle en $\mathrm{mm}$. 5 : paraglosse, face ventrale $\cdot 6:$ bord postérieur du fémur, dans la région moyenne $-7:$ griffe tarsale et denticulations (a : vue de profil ; $b$ : autre exemplaire en vue oblique) -8 : ornementation cuticulaire et bord postérieur du $6^{e}$ tergite abdominal - 9 : paraprocte -10 : paracerque et départ des cerques. 
Pattes claires, hormis les apex brun foncé des fémurs et des tarses. Une maculation brune, allongée, peu visible, dans la région moyenne des fémurs. assez près du bord postérieur. Par rapport à $B$. alpinus, ce bord postérieur porte (fig. 6) des soies plus épaisses, brusquement coniques à l'extrémité et par ailleurs moins nombreuses, de même que les grosses épines marginales, disposées sur un seul rang à l'exception de quelques isolées. Griffes tarsales (fig. 7) portant 2 soies subapicales et 8 à 11 (rarement 12 ou 13) denticulations très développées - en particulier la première - sur la face interne.

\section{ABDOMEN.}

Maculations sur les tergites très comparables à celles de $\boldsymbol{B}$. alpinus mais moins marquées. La couleur de fond des tergites est brun jaunâtre clair assez uniforme. Le bord postérieur des tergites (fig. 8) porte de fortes denticulations, comparables à celles de $B$. alpinus, mais la surface tergale paraît dépourvue d'écailles et rappelle plutôt celle de $B$. melanonyx, avec de fortes impressions concaves (" semilunar impressions ").
7 paires de branchies, généralement plus développées que chez $B$. alpinus: la longueur des $4^{c}$ ou $5^{c}$ atteint souvent les longueurs cumulées de 2 tergites consécutifs. Leur contour, nettement asymétrique, est très peu pigmenté.

Paraproctes (fig.) portant quelques fortes épines marginales et une ou deux grosses écailles submarginales arrondies.

Cerques et paracerque jaune grisâtre. Les cerques sont un peu plus longs que le corps chez le or et, au contraire, un peu plus courts chez la $\subsetneq$. Le paracerque compte de 15 à 25 segments environ (fig. 10).

\section{TAILlE.}

Longueur du corps : 5,9 à $6,9 \mathrm{~mm}$ pour le $0,7,0$ à $8,6 \mathrm{~mm}$ pour la $\$$; des cerques : 6,4 à $8,0 \mathrm{~mm}$ pour le $\sigma^{*}, 6,2$ à $7,8 \mathrm{~mm}$ pour la $९$; du paracerque : 0,4 à $0,9 \mathrm{~mm}$.

\section{2. - Baetis maurus Kimmins, 1938}

Dans notre dernière publication (Soldán et Thomas, 1983), nous avons redécrit Baetis iberi Navás,

\begin{tabular}{|c|c|c|}
\hline & E. puntions & 5. Matrus \\
\hline \multicolumn{3}{|l|}{ - LABRE : } \\
\hline - contour apical & & Plus convexe et plus incisế au mil ỉeu \\
\hline - grandes saies frontales & $1+11-12(10-14)$ & $1+8-11$ \\
\hline - APEX DU PALPE MAXILLAIRE & $\begin{array}{l}\text { Ecailles articulées et/ou denti- } \\
\text { culations bien visibles }\end{array}$ & $\begin{array}{l}\text { o écaille articulée ; denticula- } \\
\text { tion ( } 1 \text { en général) plus faible }\end{array}$ \\
\hline - PROSTHECAS DROITE ET GAUCHE & & Beaucoup plus asymétriques \\
\hline \multicolumn{3}{|l|}{ - PALPE LABIAL : } \\
\hline $\begin{array}{l}\text { - rapport Longueur/largeur } \\
\text { des } 2 \text { e et 3e articles }\end{array}$ & $\simeq 2,2$ & $\approx 2,9$ \\
\hline - forme du 3e article & & Plus triangulaire \\
\hline - PARAGLOSSES & & Soies apicales bien plus nombreuses \\
\hline $\begin{array}{l}\text { - DENT ICULATIONS SOUS LES } \\
\text { GRIFFES TARSALES }\end{array}$ & 1 rangēe & 2 rangëes \\
\hline - SURFACE DES TERGITES & $\begin{array}{l}\text { Nombreuses grandes impressions } \\
\text { concaves ("semi lunar impres sions") }\end{array}$ & Pas de telles impressions \\
\hline - PARAPROCTES & $\begin{array}{l}\text { Essentiel lement grandes dents mar- } \\
\text { ginales non articulées }\end{array}$ & Ecailles sous marginales articulées \\
\hline - PARACERQUE & $15-25$ articies & $6-8$ articles \\
\hline
\end{tabular}


1913, en nous fiant essentiellement au dessin de Navás relatif aux ailes postérieures. Le D: J. Alba Tercedor nous a informés, après examen de l'holotype de $B$. iberi, que cet te espèce est synonyme de B. rhodani Pictet. Nos dessins et notre description s'appliquent en réalité à l'espèce, peu connue jusqu'ici, B. pavidus Grandi 1949, très commune en Tunisie et en Algérie. B. maurus Kimmins est une espèce valide (voir Alba Tercedor, 1983, et les données du présent travail) mais, en Afrique du Nord, son aire de répartition est probablement limitée au Maroc et à l'Ouest de l'Algérie.

Le tableau p. 110 met en évidence les principales différences morphologiques observées entre B. punicus et B. maurus Kimmins (1938). Cet te dernière espèce vient par ailleurs d'être redécrite (Alba Tercedor, 1983).

\section{3. - Matériel examiné et répartition}

a) B. punicus

15 larves au dernier stade $(9 \circ, 6$ 9) provenant de l'Oued Ennour, petit affluent de l'Oued El Kébir, à $400 \mathrm{~m}$ d'altitude (près d'Ain Draham, province de Jendouba, au Nord-Ouest de la Tunisie) et récol tées le 14 avril 1982 (M. Boumaiza leg.).

L'espèce paraît très localisée et n'a, pour l'instant, pas été trouvée dans d'autres cours d'eau tunisiens.

L'holotype et 10 paratypes sont déposés au laboratoire d'Hydrobiologie de Toulouse, 2 paratypes à l'institut d'Entomologie de Ceské Budejovice, 2 autres au laboratoire de Biologie Animale de Tunis.

Ce matériel a été comparé à des larves de B. alpinus des Pyrénées. Description de référence de cette espèce : Müller-Liebenau (1969).

\section{b) B. maurus}

Plusieurs dizaines de larves au dernier stade, capturées entre le 4 et le 22 mai 1981, provenant d'affluents des oueds Isser et Tafna (Nord-Ouest de l'Algérie, Wilaya de Tlemcen).

Cette citation est nouvelle pour la faune de l'Algérie.

Nous avons plaisir à remercier M.J. Gagneur (Université de Tlemcen) pour le legs de ce matériel.

\section{Travaux cltés}

Alba Tercedor (J.). 1983. - Baetis maurus Kimmins, 1938 (Ephemeroptera : Baetidae) en la Peninsula Ibèrica. Aportación al conocimiento morfológico de las ninfas. Boln Asoc. esp. Ent., 6 (2): $173-178$.

Müller-Liebenau (T.). 1969. - Revision der europäischen Arten der Gattung Baetis Leach, 1815 (Insecta, Ephemeroptera). Gewäss. Abwäss., $48.49: 1.214$.

Soldan (T.) et Thomas (A.G.B.). 1983. - New and little-known species of mayflies (Ephemeroptera) from Algeria. Acta ent. bohemoslov., $80(5): 356 \cdot 376+2 \mathrm{pl}$.

Thomas (A.G.B.) et Gazagnes (G.). 1983. - Ephéméroptères du SudOuest de la France. III. Baetis nicolae n. sp. des Pyrénées (Baetidae). Bull. Soc Hist. nat. Toulouse. $119(1-4)$ : sous presse. 